\title{
Antegrade balloon occlusion of inferior vena cava during thrombectomy for renal cell carcinoma
}

\author{
Charles Metcalfe, MD; ${ }^{*}$ Laura Chang-Kit, MD; ${ }^{*}$ Ioana Dumitru, MD; ${ }^{\dagger}$ Shaun MacDonald, MD; ${ }^{\ddagger}$ Peter Black, MD, \\ FRCSC ${ }^{*}$
}

\begin{abstract}
Nephrectomy with inferior vena cava (IVC) thrombectomy for advanced renal cell carcinoma (RCC) is a challenging and morbid surgical case. We describe the use of a simple endoluminal technique to occlude the suprahepatic IVC during thrombectomy. A 60-year-old male presented with a large right-sided RCC and IVC tumour thrombus. The tip of the thrombus, which was nonadherent to the caval wall, extended to the level of the hepatic veins. After complete dissection of the kidney, we obtained suprahepatic control of the IVC by a large compliant balloon, introduced through the right internal jugular vein and inflated just below the level of the diaphragm. The IVC thrombectomy was performed in a bloodless field. Mean blood pressure remained stable during IVC balloon inflation with a total occlusion time of 10 minutes. Intraprocedural completion cavogram and postoperative Doppler ultrasonography showed no residual IVC clot. Blood loss during the thrombectomy portion of the case was scant. The patient's postoperative course was uncomplicated and, at the last followup, he had stable metastatic disease on sunitinib therapy. For the surgical treatment of RCC with retrohepatic IVC tumour extension, transjugular balloon occlusion of the suprahepatic IVC offers an alternative to extensive hepatic mobilization to obtain suprahepatic thrombus control. Advantages over traditional surgical methods may include decreased surgical time, lower risk of liver injury and tumour embolism. We suggest this method for further evaluation.
\end{abstract}

Can Urol Assoc J 2010;4(4):E105-108

\section{Introduction}

About $4 \%$ to $10 \%$ of patients with renal cell carcinoma (RCC) will present with thrombus in the inferior vena cava (IVC). ${ }^{1}$ Aggressive surgical resection with radical nephrectomy and IVC thrombectomy result in cure in about $45 \%$ to $70 \%$ of these patients. ${ }^{2}$ The level of the thrombus is generally described according to the Neves and Zincke classification. ${ }^{3}$ Thrombectomy is conducted after mobilization of the affected kidney outside of Gerota's fascia with ligation and transection of the renal arterial supply. Traditional vascular control for IVC thrombectomy consists of dissection of the involved IVC, with sequential clamping of the distal IVC, contralateral renal vein, lumbar branches and the proximal IVC cephalad to the thrombus. The technique for proximal control of the IVC depends on the level of thrombus extension. Infrahepatic control with clamps is usually adequate for level I thrombus, but mobilization of the liver and temporary occlusion of the portal triad (Pringle manoeuvre) are commonly required for level II and III thrombus. For level IV thrombus, sternotomy with cardiopulmonary bypass and an opening of the right atrium are often required. ${ }^{3}$

We present a case of a patient with level II tumour thrombus extending from a large right renal tumour, who underwent a cytoreductive nephrectomy and thrombectomy using antegrade balloon occlusion for proximal control of the IVC.

\section{Case report}

A 60-year-old male with ECOG (Eastern Cooperative Oncology Group) status 2 presented with gross hematuria. ${ }^{4}$ Computed tomography (CT) of the abdomen revealed a right renal mass measuring $9.5 \mathrm{~cm}$ in maximal diameter and extending through Gerota's fascia with multiple nodular opacities in the pararenal and perirenal space (Fig. 1). There was tumour thrombus extending into the retrohepatic IVC up to the level of the caudate lobe (Fig. 2). There was poor contrast filling in both common iliac veins, suggesting distal extension of tumour thrombus to the confluence of the common iliac veins. Metastatic evaluation revealed a $3.3 \mathrm{~cm}$ contralateral renal mass, an ipsilateral adrenal lesion and multiple pulmonary nodules up to $1.2 \mathrm{~cm}$.

After counselling the patient on his treatment options, the patient decided to proceed with cytoreductive nephrectomy and IVC thrombectomy, followed by postoperative targeted therapy. The vascular surgery service was consulted for preoperative planning, and the decision was made to proceed with antegrade balloon occlusion of the IVC for proximal control. A cardiovascular surgeon and the perfusionist were available if needed and a cardiopulmonary bypass machine was primed and ready for possible use. 


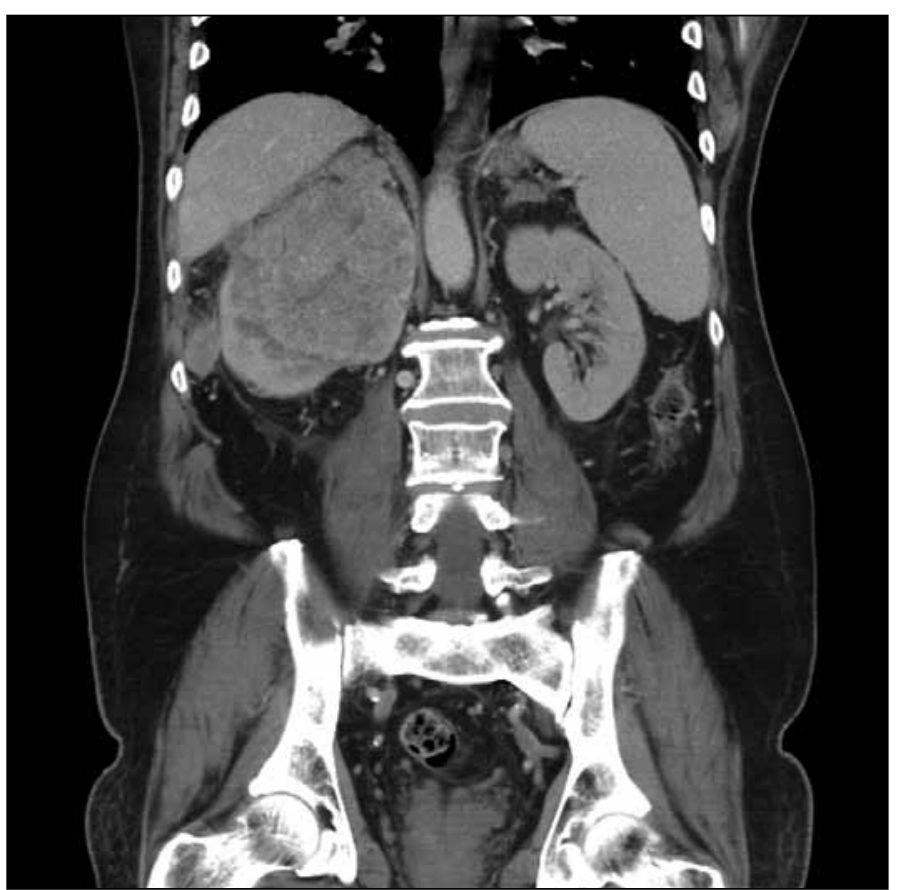

Fig. 1. Large right renal cell carcinoma $(9.4 \mathrm{~cm} \times 9.5 \mathrm{~cm})$

\section{Surgical technique}

Anesthesia was conducted with concomitant transesophageal echocardiography. A large bore left subclavian central

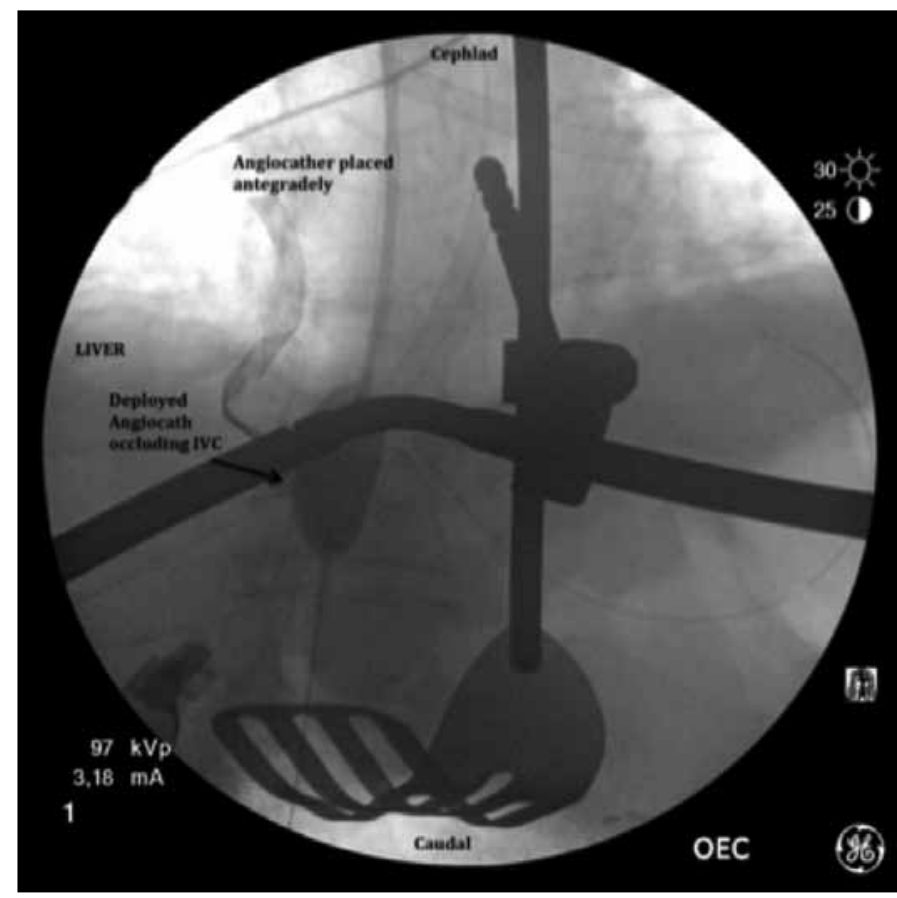

Fig. 3. Guidewire and compliant balloon catheter placement via the right internal jugular vein. Guidewire passage to the infrarenal IVC was deferred until resection of the mobile suprahepatic tumour thrombus had been achieved.

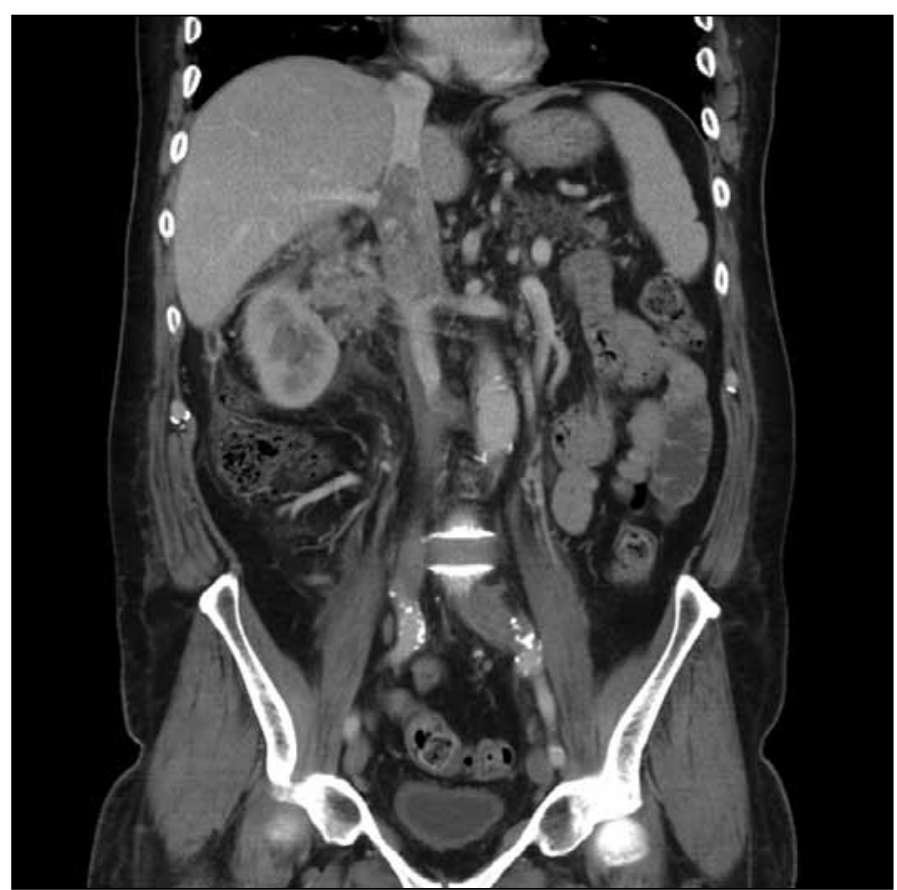

Fig. 2. Extent of IVC tumour thrombus (level II).

line was placed in order to leave the right internal jugular vein available for the intracaval balloon. The surgical site was prepared to include the area from the neck down to the mid thigh, including potential vascular access sites in the jugular, axillary and femoral regions.

The kidney and IVC were exposed through a subcostal incision carried across the contralateral rectus muscle (partial chevron). Significant collateral venous drainage was encountered around the kidney. The right renal artery was identified and transected. The kidney was dissected circumferentially, leaving the kidney attached only by the right renal vein. Rummel tourniquets were placed around the infrarenal IVC (below the palpable thrombus), two large lumbar veins and the left renal vein. The plane posterior to the suprarenal IVC was developed to allow later clamping. The mean arterial pressure had been maintained between 50 and $60 \mathrm{mmHg}$.

Under sterile conditions, using ultrasound guidance, the right internal jugular vein was cannulated and a 14 French sheath (St. Jude Medical, Minnetonka, MN) was placed. Using standard exchange techniques and fluoroscopic guidance, a 0.035 inch Rosen guidewire (Cook Medical, Bloomington, IN) was placed to the level of the tip of the tumour thrombus as determined by preoperative CT angiography imaging. A 32-mm compliant CODA balloon catheter (Cook Medical, Bloomington, IN) was advanced to the tip of the guidewire and an inflated, occluding the IVC and hepatic veins. After a few minutes of trial inflation, the patient remained hemodynamically stable with a norepinephrine infusion that had been titrated up to $8 \mu \mathrm{g} / \mathrm{min}$ and the sur- 
gical team continued with the tumour thrombectomy.

The right renal vein was entered at its confluence to the IVC. The renal vein wall was detached circumferentially and the mobile retroheptic thrombus was extracted en bloc with ring forceps. The cavotomy was temporarily clamped (Satinsky clamp, Aesculap, Center Valley, PA), and the guidewire was advanced to the proximal IVC. The intracaval balloon was repositioned below the level of the inflow of the hepatic veins. The cavotomy was unclamped and adherent thrombus was removed from the infrarenal IVC down to the confluence of the iliac veins. The cavotomy was reclamped and a cavogram was performed. Using standard angiographic techniques, we advanced a pigtail catheter to the level of the iliac vein confluence via the right internal jugular access site. The cavogram revealed residual thrombus in the infrarenal IVC. Balloon occlusion of the IVC was re-established and the remaining thrombus was removed. A second cavogram revealed no residual clot and the cavotomy was repaired. All tributary veins were released after a total of 10 minutes of IVC occlusion. A para and retrocaval node dissection were performed prior to closure of the incision. The total surgical time was 205 minutes. The estimated blood loss was $2.5 \mathrm{~L}$, which was attributed to collateral veins around the kidney and occurred mostly before entering the IVC.

\section{Postoperative course}

The patient's postoperative recovery was unremarkable. By the second postoperative day, his creatinine level had improved over the preoperative level and the liver function tests were normal. A Duplex ultrasound revealed no residual clot in the IVC, iliac veins or deep femoral veins.

The final pathology revealed a $9.5 \mathrm{~cm} \mathrm{RCC}$, clear cell type with Fuhrman nuclear grade 4 . The adrenal gland had direct invasion and the retroperitoneal lymph nodes were positive for RCC. The pathologic staging was pT4N2M1. At follow-up (6 months postoperative), the patient has stable disease on sunitinib therapy.

\section{Discussion}

Nephrectomy with IVC thrombectomy remains one of the most technically challenging and morbid procedure in urology. ${ }^{5}$ Adequate suprahepatic exposure and IVC control can be difficult and often require full mobilization of the liver or sternotomy with pericardiotomy. ${ }^{6}$ Exposure often requires a multidisciplinary collaboration with either the cardiac or vascular surgery services. ${ }^{7}$ Faced with this problem, we chose instead to employ straight forward endoluminal principles and techniques to gain suprahepatic IVC control. We found this technique to work effectively and without difficulty.

This method has been reported once previously for IVC control during RCC caval thrombectomy. Yong Yang and colleagues retrospectively reviewed data from a total of 10 patients that underwent temporary balloon occlusion of the IVC during resection of thrombus. ${ }^{8}$ Access was gained through the right internal jugular vein. Under fluoroscopic guidance, the catheter was positioned just cranial to the thrombus. A trial of occlusion was done prior to each procedure for 5 minutes, checking for significant blood pressure and heart rate changes. The technique of occulsion, in order to achieve a bloodless field during thrombectomy, was similar to our technique. One of the 10 patients was unable to tolerate temporary occlusion and therefore was converted to IVC-right atrium bypass. The remaining 9 patients tolerated the procedure well, and their circulatory status and blood pressure remained stable throughout. Mean occlusion time was 26 minutes and blood loss varied between $400 \mathrm{~mL}$ to $600 \mathrm{~mL}$ in this study.

Introduction of an occlusion balloon through the cavotomy has also been described in 13 cases of level II and III thrombus. ${ }^{9}$ Suprahepatic balloon positioning is accomplished with the aid of transesophageal echocardiography (TEE). In 1 case, tumour migration was detected by TEE during the procedure.

The principle advantage of balloon occlusion is the ability to avoid liver mobilization. This reduces surgical time, the risk of liver injury and the risk of injury to the hepatic venous branches during mobilization. ${ }^{6}$ Transjugular suprahepatic balloon occlusion allows accurate balloon positioning without the need to pass any hardware past the mobile suprarenal thrombus; this should decrease the risk of disrupting the thrombus. Balloon occlusion reduces the risk of two potentially fatal complications: tumour embolism to the pulmonary arteries and air embolism. ${ }^{10}$

The ability to easily and accurately reposition the balloon caudally after resection of the suprarenal thrombus should immediately reconstitute hepatic vein flow. Hepatic congestion occurs during occlusion over the hepatic veins, but is relieved by repositioning, and the risk of hepatic ischemia, that is otherwise associated with IVC thrombectomy, is avoided. ${ }^{6}$

An additional advantage of transjugular balloon placement is the ability to perform an intra-operative completion cavogram to ensure complete removal of the thrombus. This is well exemplified in our case, during which residual bland thrombus in the distal IVC was identified and removed. In this case, the balloon was removed to allow introduction of the pigtail catheter into the infrarenal IVC. A more elegant method would be the introduction of a 5 French sheath through the silicon diaphragm of the main sheath. This would have allowed the quick exchange of angiography catheters without the need to remove guidewire or balloon catheter.

The principle risk of balloon techniques is the potential of inadequate IVC occlusion without adequate anatomical exposure to obtain surgical control. Balloon failure, although 
Metcalfe et al.

unlikely, could be catastrophic. Surgical exposure may also be inadequate if significant IVC resection and grafting are necessary due to adherent thrombus. Furthermore, there is a very small risk of cardiac arrhythmia due to irritation of the right atrium by the wire or catheter. ${ }^{11}$ Certainly balloon placement should only be performed by an operator with refined endovascular skills. Finally, the endoluminal approach does not provide appropriate control if the thrombus extends above the hepatic veins, due to the lack of space to inflate the balloon.

Regardless of the technique for subdiaphragmatic control of the IVC, there is always the potential that the patient will not be able to tolerate the reduced venous return. While it may be a concern that endoluminal control will not allow rapid access to the heart for cardiopulmonary bypass, our planned strategy in this case would be veno-venous bypass from the femoral vein to the axillary vein without sternotomy. This was not required in the case described, but the inclusion of vascular surgeons with appropriate expertise will make this a feasible surgical approach when needed.

\section{Conclusion}

Transjugular balloon occlusion of the suprahepatic IVC is a safe and efficient method for control of the IVC during IVC thrombectomy. The balloon can be readily positioned at the level of the hepatic veins and moved inferiorly as needed. This method also allows one to perform a cavogram to ensure complete thrombus removal. In carefully selected patients, this procedure has considerable potential advantages over other standard methods and should reduce the morbidity for the patient.

*Department of Urological Sciences, UBC, Vancouver, BC; †Department of Pharmacology and Anesthesiology, St Paul's Hospital, UBC, Vancouver, BC; ‡Department of Surgery, St. Paul's Hospital, $\mathrm{UBC}$, Vancouver, $\mathrm{BC}$
Competing interests: None declared.

This paper has been peer-reviewed.

\section{References}

1. Pagano F, Dal Bianco M, Artibani $W$, et al. Renal cell carcinoma with extension into the inferior vena cava: Problems in diagnosis, staging and treatment. Eur Urol 1992;22:200-3.

2. Wein AJ, Kavoussi LR, Novick AC, Partin AW, Peters CA, editors. Campbell-Walsh Urology. 9th edition. Philadelphia, PA: Saunders; 2007:table 47-15.

3. Blute ML, Leibovich $B C$, Lohse CM, et al. The Mayo Clinic experience with surgical management, complications and outcome for patients with renal cell carcinoma and venous tumour thrombus. BJU Int 2004:94:33-41.

4. Jibiki M, Iwai $T$, Inoue Y, et al. Surgical strategy for treating renal cell carcinoma with thrombus extending into the inferior vena cava. J Vasc Surg 2004;39:829-35.

5. Oken MM, Creech RH, Tormey DC, et al. Toxicity and response criteria of the Eastern Cooperative Oncology Group. Am I Clin Oncol 1982;5:649-55.

6. Ciancio $G$, Vaidya $A$, Savoie $M$, et al. Management of renal cell carcinoma with level III thrombus in the inferior vena cava. J Urol 2002;168(4Pt1):1374-7.

7. Gallucci $M$, Borzomati $D$, Flammia $G$, et al. Liver harvesting surgical technique for the treatment of retrohepatic caval thrombosis concomitant to renal cell carcinoma: perioperative and long-term results in 15 patients without mortality. Eur Urol 2004;45:194-202.

8. Yamashita C, Azami T, Okada M, et al. Usefulness of cardiopulmonary bypass in reconstruction of inferior vena cava occlusion by renal cell carcinoma tumour thrombus. Angiology 1999:50:47-53.

9. Yang Y, Sun S, Xiao X, et al. Temporary balloon occlusion of inferior vena cava in resection of renal Tumour with vena cava thrombus extension. Urology 2009;73:645-8.

10. Zini L, Haulon S, Leroy $X$, et al. Endoluminal occlusion of the inferior vena cava in renalcell carcinoma with retro- or suprahepatic caval thrombus. BJU Int 2006;97:1216-20.

11. Sigman DB, Hasnain JU, Del Pizzo JJ, et al. Real-time transesophageal echocardiography for intraoperative surveillance of patients with renal cell carcinoma and vena caval extension undergoing radical nephrectomy. J Urol 1999;161:36.

12. Grech E. ABC of interventional cardiology. BMJ 2003;326:1137-40.

Correspondence: Dr. Peter C. Black, Department of Urologic Sciences, UBC, Level 6, 2775 Laurel St., Vancouver, BC V5Z 1M9; fax: 604-875-5677; peter.black@ubc.ca 